\title{
AQUEOUS EXTRACT OF MUSTARD CROP RESIDUES ON WEED MANAGEMENT AND CROP PERFORMANCE OF WHEAT
}

\author{
D. Sarkar, M.R. Uddin*, S.K. Paul, U.K. Sarker, \\ F. Ahmed, S. Pramanik and D.N. Chadny \\ Department of Agronomy \\ Bangladesh Agricultural University \\ Mymensingh-2202, Bangladesh
}

\begin{abstract}
Allelopathy is expected to be an important mechanism in the plant invasion process. The experiment was conducted at the Agronomy Field Laboratory, Bangladesh Agricultural University, Mymensingh to evaluate the effect of aqueous extract of mustard crop residues on weed management and crop performance of wheat. The experiment consisted of three cultivars of wheat viz., BARI Gom 19, BARI Gom 21, BARI Gom 24 and five levels of aqueous extract such as no crop residues $\left(\mathrm{C}_{0}\right)$, mustard crop residues: chaffed leaves soaked in water in 1:20 ratio $(w / v)\left(C_{1}\right), 1: 30$ ratio $(w / v)\left(C_{2}\right)$, $1: 40$ ratio $(\mathrm{w} / \mathrm{v})\left(\mathrm{C}_{3}\right)$, hand weeding $\left(\mathrm{C}_{4}\right)$. The maximum weed growth was noticed with the cultivar BARI Gom 19 and the minimum was found in the cultivar BARI Gom 21. The grain yield as well as the other yield contributing characters produced by BARI Gom 21 was the highest among the studied varieties. The highest percent inhibition of all weed species was caused by hand weeding. The highest numbers of tillers hill $^{-1}$, numbers of grains spike ${ }^{-1}, 1000$ grain weight, grain yield and straw yield were observed where hand weeding is done and followed by the application of aqueous extract in 1:20 ratio $(w / v)$. BARI Gom 21 cultivar with all treatments produced the highest grain and straw yield among the treatment combination. The results indicate that different amount of aqueous extract of mustard crop residues showed potential activity to suppress weed growth and it has a significant effect on the yield of wheat. Therefore, mustard crop residues might be used as an alternative way for weed management in effective and sustainable crop production.
\end{abstract}

Keywords: Wheat varieties, Weed population, Inhibition, Mustard crop residues, Yield, Harvest index.

*Corresponding author: romijagron @ bau.edu.bd 


\section{INTRODUCTION}

Bangladesh is an agrarian economy where total area under wheat cultivation is 4.288 lakh hectares with an annual production of 14.236 lac m. tons during 2016-2017 and an average yield of $3.320 \mathrm{t} \mathrm{ha}^{-1}$ (AIS, 2018). Poor stand establishment and heavy weed infestation during the growing stage are major obstacles to the large-scale adoption of wheat. When the weeds and crop emerge at the same time, yield losses generally increase several-fold (Aldrich, 1987). Weeds exhibit the economic yield losses to the wheat crop, which may range from $24-39.5 \%$ and these must be controlled during the full growing season of the crop for achieving satisfactory crop yields (Oad et al., 2007).

As such, the productivity and profitability of wheat solely rely on weed management. Heavy use of herbicides may also result in the evolution of herbicideresistant weeds (Duke et al., 2001). The reliance on synthetic herbicides which otherwise may cause environmental and health-related problems, as well as the evolution of herbicide-resistant weeds in the near future. The evolution of resistance in weeds against the synthetic herbicides and the presence of miscellaneous weeds (flora) in wheat have forced scientists to find eco-friendly alternatives to managing weeds in this wheat production system.

During the last two decades, researchers have focused on organic and environmentally friendly approaches to weed management to replace synthetic herbicide application (Jabran et al., 2015) in various crops. The incidence of growth inhibition of certain weeds and the induction of phytotoxic symptoms by plants and their residues is well documented for many crops, including all major grain crops such as rice, rye, barley, sorghum, wheat, mustard, marsh pepper, hairy vetch, buckwheat and other crop residues (Uddin et al., 2010; Won et al., 2011; Uddin et al., 2012, 2014; Ahmed et al., 2018; Pramanik et al., 2019; Sarker et al., 2020). Crop residues can interfere with weed development and growth through alteration of soil physical, chemical, and biological characteristics. Allelochemicals from several plants have been identified and their activities have also been established. Plant water extracts have been tested and found effective for weed control in several field crops (Cheema et al., 1997, 2001, 2002; Wazir et al., 2011). Other allelopathic weed management strategies for weed control in various crops may involve crop mulches (Cheema et al., 2000; Sarker et al., 2020), soil incorporation of crop residues (Matloob et al., 2010), or the inclusion of crops with allelopathic potential in crop rotations (Einhellig and Rasmussen, 1989). Mustard is a successful competitor against weeds in fields as empirically known (Hossain et al., 2017). However, in Bangladesh, so far, a little attempt has been made to exploit the allelopathy of plants for possible weed control purposes in the agriculture sector. So, the study was conducted to investigate the weed-suppressing ability and to determine the optimum dose of aqueous extract of mustard crop residues for the establishment of an easy, economical and sustainable method for efficient weed management and better yield of wheat. 


\section{MATERIALS AND METHODS}

The experiment was carried out at the Agronomy Field Laboratory of Bangladesh Agricultural University, Mymensingh from November 2016 to March 2017, located at $24^{\circ} 75^{\prime} \mathrm{N}$ latitude and $90^{\circ} 50^{\prime} \mathrm{E}$ longitude at an elevation of $18 \mathrm{~m}$ above the mean sea level characterized by non-calcareous dark grey floodplain soil belonging to the Old Brahmaputra Floodplain, (AEZ-9). The climate is humid subtropical monsoon. The physicochemical properties of the soil before the beginning of the experiment are shown in Table1.

Table 1. Physicochemical properties of soil before start of the experiments

\begin{tabular}{lc}
\hline Soil texture & Clayloam \\
\hline $\mathrm{pH}-\mathrm{H} 2 \mathrm{O}$ & 5.83 \\
Ec $(\mu \mathrm{s} / \mathrm{cm})$ & 143 \\
Organic carbon $(\%)$ & 1.125 \\
Total N $(\%)$ & 0.145 \\
Available P $(\mathrm{ppm})$ & 23.3 \\
Available K $(\mathrm{ppm})$ & 88.64 \\
Available S $(\mathrm{ppm})$ & 59.64 \\
\hline
\end{tabular}

The experiment consists of two factors including crop residues (5) i) no use of extract (control), ii) aqueous extract of mustard crop residues @ 1:20 ratio (w/v), iii) aqueous extract of mustard crop residues @ 1:30 ratio (w/v), iv) aqueous extract of mustard crop residues@1:40 ratio (w/v) and v) hand weeding and variety (3): i) BARI Gom19 ii) BARI Gom21 iii) BARI Gom 24. The seeds were sown on 21 November 2016 as per treatment specifications. The experiment was laid out in a randomized complete block design with three replications. The small pieces of sorghum crop residues were dipped into water for 24 hours and then collected the aqueous extract from residues. The prepared sorghum aqueous extract was applied two times (20 days and 40 days) after seed sowing by a hand sprayer and harvesting was done at 3 months 15 days after sowing. Data were collected on the basis of different parameters of wheat and weeds. Among them percent inhibition shows the suppressing ability of aqueous extract of sorghum residues on weed.

Inhibition $(\%)=\frac{\text { Dry weight of weed at control-Dry weight of weed from treatment }}{\text { Dry weight of weed at control }} \times 100$

Data were also collected from wheat on yield basis such as grain yield, straw yield, harvest index, etc., which showed the yield performance of wheat. The recorded data were compiled and tabulated for statistical analysis. Analysis of variance was done with the help of a computer package, MSTAT-C program. The mean differences among the treatments were adjudged by Duncan's Multiple Range Test. 


\section{RESULTS AND DISCUSSION}

\section{Infested weed species in the experimental field}

Seven weed species belonging to five families infested the experimental field. Local name, scientific name, family, morphological type and life cycle of the weed in the experimental plot have been presented in Table 1. Ahmed and Uddin (2018) also reported some major weeds infestation in wheat field and their effective suppression by the application of sorghum crop residues.

Table 2. Infested weed species found growing in the experimental plots in wheat

\begin{tabular}{llllll}
\hline $\begin{array}{l}\text { S1. } \\
\text { No. }\end{array}$ & $\begin{array}{l}\text { Local } \\
\text { name }\end{array}$ & Scientific name & Family & $\begin{array}{l}\text { Morphological } \\
\text { type }\end{array}$ & Life cycle \\
\hline 1 & Bathua & Chenopodium album & Chenopodiaceous & Broad-leaved & Annual \\
2 & Mutha & Cyperus rotundas & Cyperaceae & Sedge & Perennial \\
3 & Durba & Cynodon dactylon & Gramineae & Grass & Perennial \\
4 & Shama & Echino chloacrusgalli & Gramineae & Grass & Annual \\
5 & Tit begun & Solanum torvum & Solanaceae & Broad-leaved & Perennial \\
6 & Biskatali & Polygonum hydropiper & Polygonaceae & Broad-leaved & Annual \\
7 & Angta & Paspalum scrobiculatum & Gramineae & Grass & Annual \\
& & & & & \\
\hline
\end{tabular}

\section{Effect of variety on number and percent inhibition on different weeds}

Variety shows a significant effect on number of weed populations for all weed species. The lowest number of weeds was found in different varieties for different weeds (Table 2). On the other hand, percent inhibition was significantly affected by variety for all weed species. Bathua (Chenopodium album), mutha (Cyperus rotundas), durba (Cynodon dactylon), biskatali (Polygonum hydropiper) and angta (Paspalum scrobiculatum) were showed 53.02, 49.29, 53.00, 52.90, $56.46 \%$ percent inhibition, respectively, for $\mathrm{V}_{2}$ and tit begun (Solanum torvum), shama (Echino chloacrusgalli) were found $57.11,55.03$ in $\mathrm{V}_{3}$ variety (Table 2). The percent inhibition of weed is significantly influenced by variety of transplanted Aman rice and residual effect of marsh pepper (Pramanik et al., 2019).

Table 3. Effect of variety on number and percent inhibition on different weeds

\begin{tabular}{|c|c|c|c|c|c|c|c|c|c|c|c|c|c|}
\hline \multicolumn{7}{|c|}{ Number of weed per quadrate $(25 \times 25) \mathrm{cm}^{2}$} & \multicolumn{7}{|c|}{ \% Inhibition } \\
\hline $\begin{array}{l}\text { Weed } \\
\text { name }\end{array}$ & Bathua & Mutha & Biskata & li Durba & $\begin{array}{l}\text { Tit Shama } \\
\text { begun }\end{array}$ & Angta & Bathua & Mutha & Biskata & li Durba & $\begin{array}{l}\text { Tit } \\
\text { begun }\end{array}$ & Shama & Angta \\
\hline $\begin{array}{l}\text { variety } \\
\mathrm{V}_{1}\end{array}$ & $3.00^{\mathrm{a}}$ & $4.33^{\mathrm{a}}$ & $7.60^{\mathrm{a}}$ & $4.40^{\mathrm{a}}$ & $3.60^{\mathrm{a}} \quad 4.27^{\mathrm{a}}$ & $3.72^{\mathrm{a}}$ & $46.37^{\mathrm{b}}$ & $39.07^{\mathrm{b}}$ & $42.94^{\mathrm{c}}$ & $41.30^{\mathrm{b}}$ & $50.83^{b}$ & $48.82^{\mathrm{b}}$ & $52.74^{\mathrm{c}}$ \\
\hline $\mathrm{V}_{2}$ & $2.53^{\mathrm{b}}$ & $2.93^{\mathrm{c}}$ & $4.27^{\mathrm{c}}$ & $2.534^{\mathrm{c}}$ & $2.65^{\mathrm{b}} 3.60^{\mathrm{b}}$ & $2.07^{\mathrm{c}}$ & $53.02^{\mathrm{a}}$ & $49.29^{\mathrm{a}}$ & $52.90^{\mathrm{a}}$ & $53.00^{\mathrm{a}}$ & $52.85^{\mathrm{at}}$ & $52.77^{\mathrm{ab}}$ & $56.46^{\mathrm{a}}$ \\
\hline $\mathrm{V}_{3}$ & $3.00^{\mathrm{a}}$ & $3.47^{\mathrm{b}}$ & $5.94^{\mathrm{b}}$ & $3.20^{\mathrm{b}}$ & $2.10^{\mathrm{c}} 3.13^{\mathrm{c}}$ & $2.74^{\mathrm{b}}$ & $51.49^{\mathrm{a}}$ & $46.26^{\mathrm{a}}$ & $48.41^{\mathrm{b}}$ & $50.95^{\mathrm{a}}$ & $57.11^{\mathrm{a}}$ & $55.03^{\mathrm{a}}$ & $54.14^{\mathrm{b}}$ \\
\hline $\mathrm{CV}(\%)$ & 6.49 & 11.64 & 5.45 & 17.08 & 10.695 .08 & 15.42 & 9.86 & 13.68 & 7.79 & 10.71 & 11.33 & 10.72 & 3.05 \\
\hline
\end{tabular}

$\mathrm{V}_{1}=$ BRRI Gom 19 (Sourav), $\mathrm{V}_{2}=$ BARI Gom 21 (Shatabdi), $\mathrm{V}_{3}=$ BARI Gom 24 (Prodip) 


\section{Effect of aqueous extract of mustard on number and percent inhibition on different weeds}

Numbers of weed populations are significantly affected by the treatments for all weed species. Weeds can be suppressed by physical hindrance or by posing chemical (allelopathy) secreted by mulching of crop residues (Khaliq et al. 2015; Reddy, 2001). The lowest weed population was found in $\mathrm{C}_{4}$ treatments (Hand weeding) followed by $\mathrm{C}_{1}$ treatment (Table 3 ). The highest percent inhibition was also found in $\mathrm{C}_{4}$ treatment, which is followed by $\mathrm{C}_{1}$ treatment, where the concentration of aqueous extract of sorghum was high (1:20). Numerically 66.32, $60.98,67.52,73.90,67.65,66.40$ and $67.36 \%$ inhibition were found in bathua $(C$. album), mutha $(C$. rotundus), biskatali ( $P$. hydropiper), durba $(C$. dactylon), tit begun ( $S$. torvum), shama (E. crusgali) and angta ( $P$. scrobiculatum) respectively, for $\mathrm{C}_{1}$ treatment (Table 3).

Table 4. Effect of aqueous extract of mustard crop residues on number and percent inhibition on different weeds

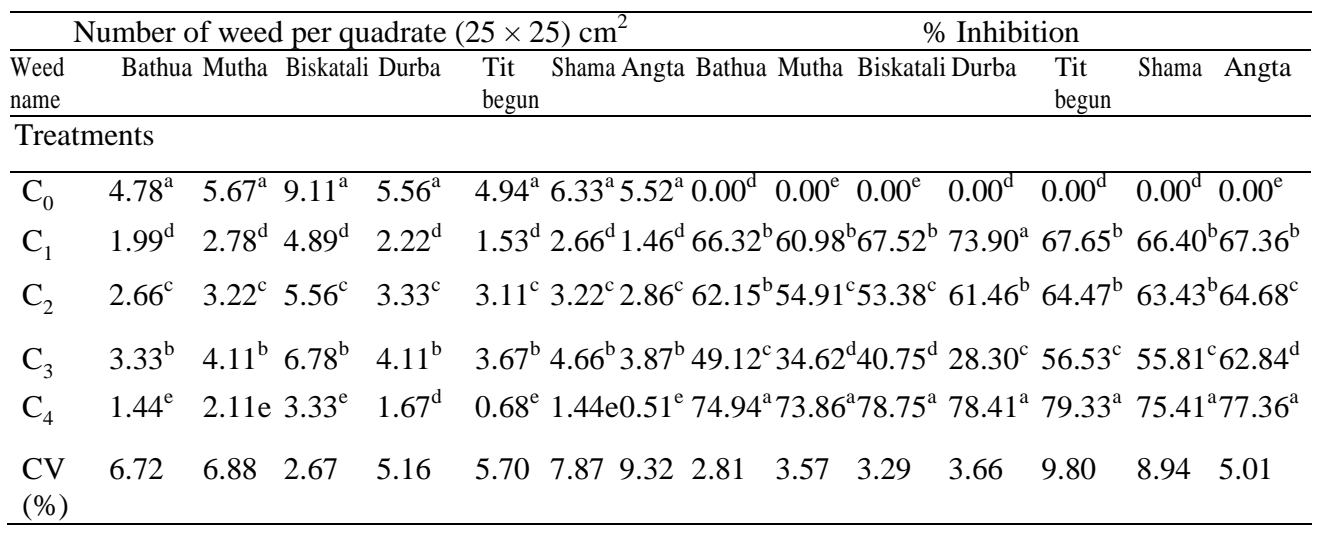

$\mathrm{C}_{0}=$ No use of extract, $\mathrm{C}_{1}=$ Aqueous extract of mustard crop residues @ 1:20 ratio (w/v), $\mathrm{C}_{2}$ $=$ Aqueous extract of mustard crop residues @ 1:30 ratio (w/v), $\mathrm{C}_{3}=$ Aqueous extract of mustard crop residues @ 1:40 ratio (w/v), $\mathrm{C}_{4}=$ Hand weeding, In a column, figures with the same letter do not differ significantly as per DMRT.

\section{The combined effect of variety and aqueous extract of mustard on number and percent inhibition on different weeds}

Numbers of weed populations are significantly affected by the combined effect of variety and treatments for all weed species except durba (Cynodon dactylon). On the other hand, highest percent inhibition was also found in the $\mathrm{V}_{2} \mathrm{C}_{4}$ combination (Table 5). 
Table 5. Combined effect of variety and aqueous extract of mustard on number and percent inhibition on different weeds

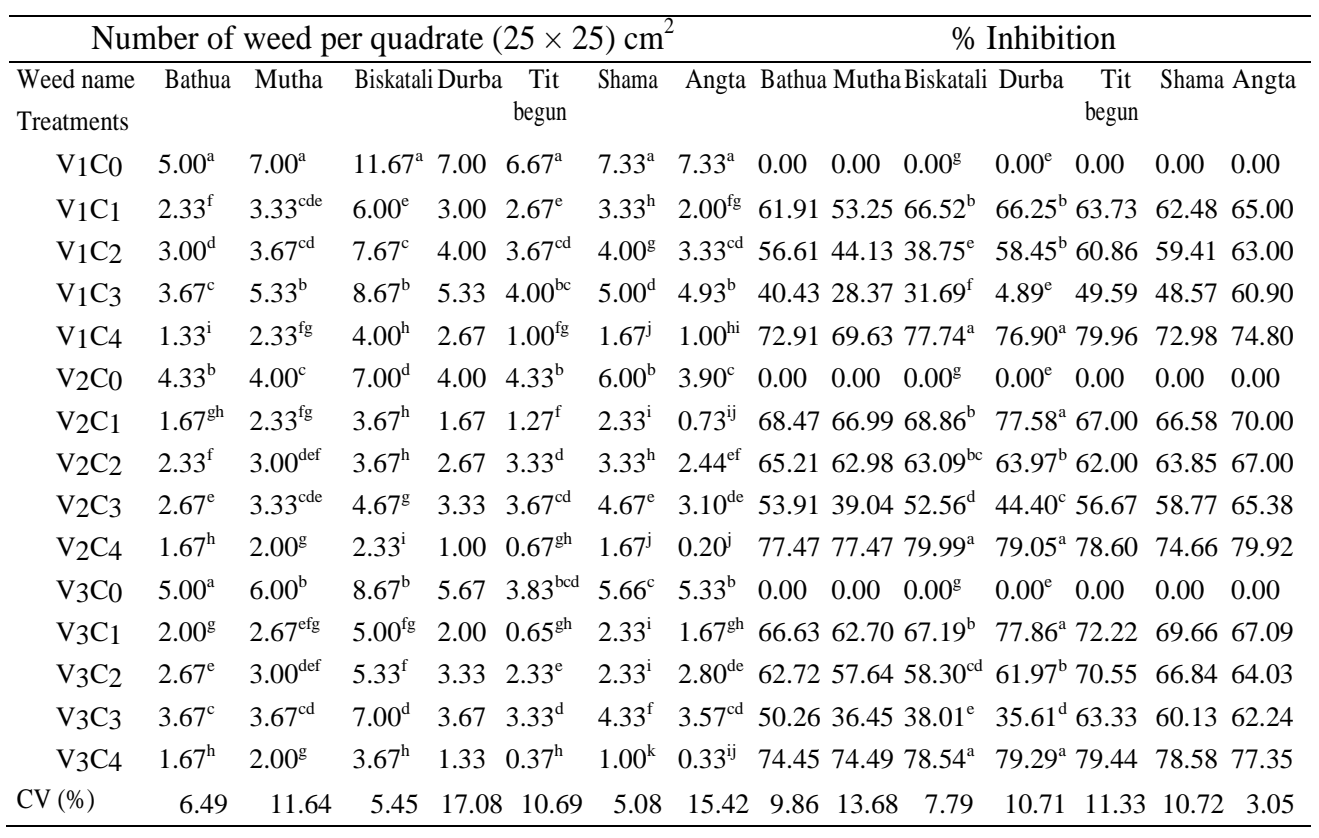

$\mathrm{V}_{1}=$ BRRI Gom 19 (Sourav), $\mathrm{V}_{2}=$ BARI Gom 21 (Shatabdi), $\mathrm{V}_{3}=$ BARI Gom 24 (Prodip), $\mathrm{C}_{0}=$ No use of extract, $\mathrm{C}_{1}=$ Aqueous extract of mustard crop residues @ 1:20 ratio (w/v), $\mathrm{C}_{2}$ $=$ Aqueous extract of mustard crop residues @ 1:30 ratio (w/v), $\mathrm{C}_{3}=$ Aqueous extract of mustard crop residues @ 1:40 ratio (w/v), $\mathrm{C}_{4}=$ Hand weeding, In a column, figures with the same letter do not differ significantly as per DMRT.

\section{Effect of variety on yield and yield contributing characters of wheat}

Varietal effect on yield and yield contributing characters of wheat showed a significant effect. Highest plant height, higher number of total tillers and effective tillers hill ${ }^{-1}$, higher number of grain spike ${ }^{-1}$, higher number of filled grain spike ${ }^{-1}$, highest straw yield and highest harvest index was found in $\mathrm{V}_{2}$ (BARI Gom21) variety (Table 5). The highest grain yield (4.08 tha $\left.{ }^{-1}\right)$ was obtained in BARI GOM21 followed by BARI GOM24 (3.87 tha $^{-1}$ ) (Fig. 1). It is reported that variety significantly differed in respect of grain yield and BR11showed the highest yield 4.09 tha $^{-1}$ (Hossain et al., 2017). 
Table 6. Effect of variety on yield and yield contributing characters of wheat

\begin{tabular}{|c|c|c|c|c|c|c|c|c|c|c|c|}
\hline Cultivars & $\begin{array}{l}\text { Plant } \\
\text { height } \\
(\mathrm{cm})\end{array}$ & $\begin{array}{l}\text { No. of } \\
\text { total } \\
\text { tillers } \\
\text { hill }^{-1}\end{array}$ & $\begin{array}{l}\text { No. of } \\
\text { effecti } \\
\text { ve } \\
\text { tillers } \\
\text { hill }^{-1} \\
\end{array}$ & $\begin{array}{l}\text { No. of } \\
\text { non } \\
\text { effectiv } \\
\text { e tillers } \\
\text { hill }^{-}\end{array}$ & $\begin{array}{l}\text { Spike } \\
\text { length } \\
(\mathrm{cm})\end{array}$ & $\begin{array}{l}\text { No. of } \\
\text { grains } \\
\text { spike }^{-1}\end{array}$ & $\begin{array}{l}\text { Filled } \\
\text { grain } \\
\text { spike }^{-1}\end{array}$ & $\begin{array}{l}1000 \\
\text { grain } \\
\text { weight } \\
(\mathrm{gm})\end{array}$ & $\begin{array}{l}\text { Grain } \\
\text { yield } \\
\text { (t ha }{ }^{-} \\
\left.{ }^{1}\right)\end{array}$ & $\begin{array}{l}\text { Stra } \\
w \\
\text { yield } \\
\left(\mathrm{t} \mathrm{ha}^{-}\right. \\
\left.{ }^{\mathrm{l}}\right) \\
\end{array}$ & $\begin{array}{l}\text { Harve } \\
\text { st } \\
\text { index } \\
(\%)\end{array}$ \\
\hline $\mathrm{V}_{1}$ & $93.59^{\mathrm{b}}$ & $4.05^{\mathrm{b}}$ & $3.46^{\mathrm{C}}$ & 0.59 & 10.30 & $42.61^{b}$ & $14.54^{\mathrm{b}}$ & 54.87 & $3.49^{\mathrm{c}}$ & $4.19^{\mathrm{c}}$ & $45.41^{\mathrm{a}}$ \\
\hline $\mathrm{V}_{2}$ & $97.13^{\mathrm{a}}$ & $4.30^{\mathrm{a}}$ & $3.72^{\mathrm{a}}$ & 0.58 & 10.71 & $44.49^{\mathrm{a}}$ & $15.35^{\mathrm{a}}$ & 55.61 & $4.08^{\mathrm{a}}$ & $5.03^{\mathrm{a}}$ & $44.78^{\mathrm{b}}$ \\
\hline $\mathrm{V}_{3}$ & $95.88^{\mathrm{ab}}$ & $4.15^{\mathrm{b}}$ & $3.58^{\mathrm{b}}$ & 0.57 & 10.48 & $43.60^{\mathrm{ab}}$ & $15.16^{\mathrm{a}}$ & 55.34 & $3.87^{\mathrm{b}}$ & $4.57^{\mathrm{b}}$ & $45.77^{\mathrm{a}}$ \\
\hline $\mathrm{CV}(\%)$ & 3.95 & 3.84 & 3.20 & 17.52 & 5.80 & 3.49 & 3.01 & 2.01 & 1.16 & 3.02 & 1.52 \\
\hline
\end{tabular}

$\mathrm{V}_{1}=$ BRRI Gom 19 (Sourav), $\mathrm{V}_{2}=$ BARI Gom 21 (Shatabdi), $\mathrm{V}_{3}=$ BARI Gom 24 (Prodip)

\section{Effect of aqueous extract of mustard crop residues on yield and yield contributing characters of wheat}

Aqueous extract of mustard crop residues had also a significant effect on yield and yield contributing characters. The highest grain yield $\left(4.22 \mathrm{tha}^{-1}\right)$ was produced by $\mathrm{C}_{4}$ treatment, followed by $\mathrm{C}_{1}\left(4.02 \mathrm{tha}^{-1}\right)$ and lowest one $\left(3.32 \mathrm{tha}^{-1}\right)$ was produced by $\mathrm{C}_{0}$ (no use of extract) treatment due to the production of higher number of effective tillers hill ${ }^{-1}$, higher number of grain spike ${ }^{-1}$, higher number of filled grain spike $^{-1}$ (Table 6 and Figure 2). Uddin and Pyon (2010) also reported the similar results, where crop residues influenced in crop performance.

Table 7. Effect of aqueous extract of mustard crop residues on yield and yield contributing characters of wheat

\begin{tabular}{|c|c|c|c|c|c|c|c|c|c|c|c|}
\hline Residues & $\begin{array}{l}\text { Plant } \\
\text { height } \\
(\mathrm{cm})\end{array}$ & $\begin{array}{l}\text { No. of } \\
\text { total } \\
\text { tillers } \\
\text { hill }^{-1}\end{array}$ & $\begin{array}{l}\text { No. of } \\
\text { effective } \\
\text { tillers } \\
\text { hill }^{-1}\end{array}$ & $\begin{array}{l}\text { No. of } \\
\text { non } \\
\text { effective } \\
\text { tillers } \\
\text { hill }^{-} \\
\end{array}$ & $\begin{array}{l}\text { Spike } \\
\text { length } \\
(\mathrm{cm})\end{array}$ & $\begin{array}{l}\text { No. of } \\
\text { grains } \\
\text { spike }^{-1}\end{array}$ & $\begin{array}{l}\text { Filled } \\
\text { grain } \\
\text { spike }^{-1}\end{array}$ & $\begin{array}{l}1000 \\
\text { grain } \\
\text { weight } \\
(\mathrm{gm})\end{array}$ & $\begin{array}{l}\text { Grain } \\
\text { yield(t } \\
\left.\text { ha }^{-1}\right)\end{array}$ & $\begin{array}{l}\text { Straw } \\
\text { yield(t } \\
\left.\text { ha }^{-1}\right)\end{array}$ & $\begin{array}{l}\text { Harv } \\
\text { est } \\
\text { index } \\
(\%)\end{array}$ \\
\hline $\mathrm{C}_{0}$ & $90.98^{\mathrm{d}}$ & $3.78^{\mathrm{e}}$ & $3.22^{\mathrm{e}}$ & 0.56 & $10.11^{\mathrm{b}}$ & $40.19^{c}$ & $13.97^{\mathrm{d}}$ & 54.73 & $3.32^{\mathrm{e}}$ & $4.05^{\mathrm{e}}$ & 45.07 \\
\hline $\mathrm{C}_{1}$ & $98.14^{\mathrm{ab}}$ & $4.39^{\mathrm{b}}$ & $3.80^{\mathrm{b}}$ & 0.59 & $10.59^{\mathrm{ab}}$ & $44.61^{\mathrm{ab}}$ & $15.39^{\mathrm{ab}}$ & 55.51 & $4.02^{\mathrm{b}}$ & $4.83^{\mathrm{b}}$ & 45.44 \\
\hline $\mathrm{C}_{2}$ & $95.89^{\mathrm{bc}}$ & $4.13^{\mathrm{c}}$ & $3.55^{\mathrm{c}}$ & 0.59 & $10.43^{\mathrm{ab}}$ & $43.86^{\mathrm{b}}$ & $15.15^{\mathrm{bc}}$ & 55.36 & $3.85^{\mathrm{c}}$ & $4.67^{\mathrm{c}}$ & 45.18 \\
\hline $\mathrm{C}_{3}$ & $92.67^{\mathrm{cd}}$ & $3.97^{\mathrm{d}}$ & $3.37^{\mathrm{d}}$ & 0.60 & $10.31^{\mathrm{b}}$ & $43.36^{\mathrm{b}}$ & $14.86^{\mathrm{c}}$ & 54.99 & $3.65^{\mathrm{d}}$ & $4.31^{\mathrm{d}}$ & 45.88 \\
\hline$\stackrel{\mathrm{C}_{4}}{ }$ & $99.98^{\mathrm{a}}$ & $4.55^{\mathrm{a}}$ & $4.008^{\mathrm{a}}$ & 0.55 & $11.04^{\mathrm{a}}$ & $45.81^{\mathrm{a}}$ & $15.70^{\mathrm{a}}$ & 55.78 & $4.22^{\mathrm{a}}$ & $5.16^{\mathrm{a}}$ & 45.04 \\
\hline $\begin{array}{l}\text { significa } \\
\text { nce }\end{array}$ & $* *$ & ** & ** & NS & * & ** & ** & NS & $* *$ & $* *$ & NS \\
\hline $\mathrm{CV}(\%)$ & 3.95 & 3.84 & 3.20 & 17.52 & 5.80 & 3.49 & 3.01 & 2.01 & 1.16 & 3.02 & 1.52 \\
\hline
\end{tabular}

$\mathrm{C}_{0}=$ No use of extract, $\mathrm{C}_{1}=$ Aqueous extract of mustard crop residues @ 1:20 ratio $(\mathrm{w} / \mathrm{v}), \mathrm{C}_{2}$ $=$ Aqueous extract of mustard crop residues @ 1:30 ratio (w/v), $\mathrm{C}_{3}=$ Aqueous extract of mustard crop residues @ 1:40 ratio (w/v), $\mathrm{C}_{4}=$ Hand weeding, $* *=$ Significant at $1 \%$ level of probability 


\section{Combined effects of variety and aqueous extract of mustard crop residues on yield and yield contributing characters of wheat}

Yield and yield contributing characters like straw yield and grain yield were significantly affected by the interaction between variety and crop residues. $\mathrm{V}_{2} \mathrm{C}_{4}$ combination showed the maximum result and the lowest result was produced by $\mathrm{V}_{1} \mathrm{C}_{0}$ combination (Table7). It is reported that BRRI dhan 56 under buckwheat crop residues $0.5 \mathrm{t} / \mathrm{ha}$ and marsh pepper residues at $1 \mathrm{t} / \mathrm{ha}$ produced the highest grain yield (Afroz et al., 2018)

Table 8. Combined effects of variety and aqueous extract of mustard crop residues on yield and yield contributing characters of wheat

\begin{tabular}{|c|c|c|c|c|c|c|c|c|c|c|c|}
\hline $\begin{array}{c}\text { Variety } \\
x \\
\text { Residue }\end{array}$ & $\begin{array}{l}\text { Plant } \\
\text { height } \\
(\mathrm{cm})\end{array}$ & $\begin{array}{c}\text { No. of } \\
\text { total } \\
\text { tillershill } \\
1\end{array}$ & $\begin{array}{c}\text { No. of } \\
\text { effective } \\
\text { tillers } \\
\text { hill }^{-1}\end{array}$ & $\begin{array}{l}\text { No. of } \\
\text { non } \\
\text { effective } \\
\text { tillers } \\
\text { hill }^{-1}\end{array}$ & $\begin{array}{l}\text { Spike } \\
\text { length } \\
(\mathrm{cm})\end{array}$ & $\begin{array}{l}\text { No. of } \\
\text { grains } \\
\text { spike }^{-1}\end{array}$ & $\begin{array}{c}\text { Filled } \\
\text { grain } \\
\text { spike }^{-1}\end{array}$ & $\begin{array}{c}1000 \\
\text { grain } \\
\text { weight } \\
(\mathrm{gm})\end{array}$ & $\begin{array}{c}\begin{array}{c}\text { Grain } \\
\text { yield } \\
\text { (tha }^{-} \\
{ }^{1} \text { ) }\end{array}\end{array}$ & $\begin{array}{c}\text { Straw } \\
\text { yield } \\
\left(\text { tha }^{-1}\right)\end{array}$ & $\begin{array}{c}\text { Harvest } \\
\text { index } \\
(\%)\end{array}$ \\
\hline $\mathrm{V}_{1} \mathrm{C}_{0}$ & 90.07 & 3.68 & 3.07 & 0.62 & 9.92 & 37.98 & $12.77^{\mathrm{e}}$ & 54.50 & $3.02^{\mathrm{i}}$ & $3.67^{j}$ & $45.20^{\mathrm{abc}}$ \\
\hline $\mathrm{V}_{1} \mathrm{C}_{1}$ & 95.80 & 4.30 & 3.73 & 0.57 & 10.40 & 44.01 & $15.19^{\mathrm{abc}}$ & 55.03 & $3.67^{\text {ef }}$ & $4.35^{\mathrm{gh}}$ & $45.76^{\mathrm{ab}}$ \\
\hline $\mathrm{V}_{1} \mathrm{C}_{2}$ & 94.87 & 4.00 & 3.37 & 0.63 & 10.22 & 43.49 & $15.17^{\mathrm{abc}}$ & 54.73 & $3.50^{\mathrm{g}}$ & $4.25^{\mathrm{gh}}$ & $45.16^{\mathrm{abc}}$ \\
\hline $\mathrm{V}_{1} \mathrm{C}_{3}$ & 90.67 & 3.87 & 3.27 & 0.60 & 10.19 & 42.87 & $14.26^{\mathrm{d}}$ & 54.57 & $3.35^{\mathrm{h}}$ & $4.00^{\mathrm{i}}$ & $45.60^{\mathrm{ab}}$ \\
\hline $\mathrm{V}_{1} \mathrm{C}_{4}$ & 96.53 & 4.40 & 3.87 & 0.53 & 10.80 & 44.71 & $15.29^{\mathrm{abc}}$ & 55.50 & $3.89^{\mathrm{d}}$ & $4.69^{\mathrm{e}}$ & $45.34^{\mathrm{abc}}$ \\
\hline $\mathrm{V}_{2} \mathrm{C}_{0}$ & 92.53 & 3.87 & 3.33 & 0.53 & 10.28 & 42.13 & $14.61^{\mathrm{cd}}$ & 55.03 & $3.62^{\mathrm{f}}$ & $4.39^{\mathrm{fg}}$ & $45.20^{\mathrm{abc}}$ \\
\hline $\mathrm{V}_{2} \mathrm{C}_{1}$ & 100.10 & 4.50 & 3.87 & 0.63 & 10.82 & 45.30 & $15.72^{\mathrm{ab}}$ & 55.90 & $4.23^{\mathrm{b}}$ & $5.35^{\mathrm{b}}$ & $44.17^{\mathrm{cd}}$ \\
\hline $\mathrm{V}_{2} \mathrm{C}_{2}$ & 97.07 & 4.27 & 3.67 & 0.60 & 10.66 & 44.39 & $15.19^{\mathrm{abc}}$ & 55.73 & $4.15^{\mathrm{c}}$ & $5.10^{\mathrm{c}}$ & $44.87^{\mathrm{bcd}}$ \\
\hline $\mathrm{V}_{2} \mathrm{C}_{3}$ & 93.87 & 4.13 & 3.53 & 0.60 & 10.38 & 43.81 & $15.21^{\mathrm{abc}}$ & 55.33 & $3.90^{\mathrm{d}}$ & $4.60^{\mathrm{ef}}$ & $45.91^{\mathrm{ab}}$ \\
\hline $\mathrm{V}_{2} \mathrm{C}_{4}$ & 102.07 & 4.73 & 4.22 & 0.52 & 11.39 & 46.82 & $16.02^{\mathrm{a}}$ & 56.07 & $4.51^{\mathrm{a}}$ & $5.79^{\mathrm{a}}$ & $43.77^{\mathrm{d}}$ \\
\hline $\mathrm{V}_{3} \mathrm{C}_{0}$ & 90.33 & 3.80 & 3.27 & 0.53 & 10.13 & 40.46 & $14.54^{\text {cd }}$ & 54.67 & $3.33^{\mathrm{h}}$ & $4.10^{\mathrm{hi}}$ & $44.82^{\text {bcd }}$ \\
\hline $\mathrm{V}_{3} \mathrm{C}_{1}$ & 98.53 & 4.37 & 3.80 & 0.57 & 10.56 & 44.51 & $15.26^{\mathrm{abc}}$ & 55.60 & $4.15^{\mathrm{c}}$ & $4.80^{\mathrm{de}}$ & $46.37^{\mathrm{a}}$ \\
\hline $\mathrm{V}_{3} \mathrm{C}_{2}$ & 95.73 & 4.13 & 3.60 & 0.53 & 10.41 & 43.71 & $15.08^{\mathrm{bcd}}$ & 55.60 & $3.89^{\mathrm{d}}$ & $4.65^{\mathrm{e}}$ & $45.51^{\mathrm{ab}}$ \\
\hline $\mathrm{V}_{3} \mathrm{C}_{3}$ & 93.47 & 3.93 & 3.33 & 0.60 & 10.35 & 43.41 & $15.10^{\mathrm{bc}}$ & 55.07 & $3.71^{\mathrm{e}}$ & $4.33^{\mathrm{gh}}$ & $46.14^{\mathrm{ab}}$ \\
\hline $\mathrm{V}_{3} \mathrm{C}_{4}$ & 101.33 & 4.53 & 3.93 & 0.60 & 10.93 & 45.89 & $15.80^{\mathrm{ab}}$ & 55.77 & $4.26^{\mathrm{b}}$ & $5.00^{\mathrm{cd}}$ & $46.01^{\mathrm{ab}}$ \\
\hline $\begin{array}{c}\text { Level of } \\
\text { significance. }\end{array}$ & NS & NS & NS & NS & NS & NS & $*$ & NS & $* *$ & $*$ & $*$ \\
\hline CV $(\%)$ & 3.95 & 3.84 & 3.20 & 17.52 & 5.80 & 3.49 & 3.01 & 2.01 & 1.16 & 3.02 & 1.52 \\
\hline
\end{tabular}

$\mathrm{V}_{1}=$ BRRI Gom19 (Sourav), $\mathrm{V}_{2}=$ BARI Gom21 (Shatabdi), $\mathrm{V}_{3}=$ BARI Gom24 (Prodip), $\mathrm{C}_{0}$ $=$ No use of extract, $C_{1}=$ Aqueous extract of mustard crop residues $@ 1: 20$ ratio $(w / v), C_{2}=$ Aqueous extract of mustard crop residues@1:30 ratio (w/v), $\mathrm{C}_{3}=$ Aqueous extract of mustard crop residues @ 1:40 ratio (w/v), $\mathrm{C}_{4}=$ Hand weeding, **=Significant at 1\% level of probability, $*=$ Significant at $5 \%$ level of probability, NS = Non significant

\section{CONCLUSION}

From the above results it was found that weed population, percent inhibition were significantly affected by variety, mustard crop residues and their interaction. The variety BARI Gom 21 with $\mathrm{C}_{4}$ (hand weeding) treatment exhibited the superior effect and BARI Gom 21 with $\left(\mathrm{C}_{4}\right)$ 1:20 ratio (w/v) showed quit close result with it. It is irrefutable that chemical control of weeds has negative impact on our natural environment whereas incorporation of mustard crop residues have pronounced influenced on yield and yield contributing characteristics of wheat showed potentiality to suppress weed growth. Indeed, application of mustard crop residues 
may be a pragmatic option for suppressing weed that emphasize optimal crop production with minimal external, reducing dependence on commercial inputs (fertilizer and pesticides) and substituting them with internal resources and relying on sustainable practices which could maintain the productivity over long periods.

\section{REFERENCES}

Afroz, F., Uddin, M.R., Hasan, A.K., Sarker, U.K., Hoque, M.M.I. and Islam, M.A. (2018). Combined allelopathic effect of buckwheat and marsh pepper residues on weed management and crop performance of transplant aman rice. Archives of Agriculture and Environmental Science, 3(3):289-296.

Ahmed, F., Uddin, M.R., Hossain, M. D., Sarker, U.K., Sarkar, D. and Chadny, D. (2018). Effect of aqueous extract of sorghum crop residues on weed management and crop performance of wheat. Bangladesh Agronomy Journal, 21(2):87-95.

AIS. (2018). Agriculture Information service, Ministry of Agriculture, Government of the People's Republic of Bangladesh. Pp. 14.

Aldrich, R.J. (1987). Predicting crop yield reductions from weeds. Weed Technology, 1:199-206.

Cheema, Z., Luqman, M. and Khaliq, A. (1997). Use of allelopathic extracts of sorghum and sunflower herbage for weed control in wheat. Journal of Animal and Plant Sciences 7:91-93.

Cheema, Z.A., Asim, M. and Khaliq, A. (2000). Sorghum allelopathy for weed control in cotton (Gossypium arboreum L.). International Journal of Agriculture and Biology, 2(1):37-41.

Cheema, Z., Khaliq, A. and Akhtar, S. (2001). Use of sorgaab (sorghum water extract) as a natural weed inhibitor in spring mungbean. International Journal of Agriculture and Biology, 3(4):515-518.

Cheema, Z., Iqbal, M. and Ahmad, R. (2002). Response of wheat varieties and some rabi weeds to allelopathic effects of sorghum water extract. International Journal of Agriculture and Biology, 4(1):52-55.

Duke, S.O., Scheffler, B.E. Dayan, F.E., Weston, L.A. and Ota, E. (2001). Strategies for using transgenes to produce allelopathic crops. Weed Technology, 15(4):826-834.

Einhellig, F.A. and Rasmussen J.A. (1989). Prior cropping with grain sorghum inhibits weeds. Journal of Chemical Ecology, 15(3):951-960.

Hossain, M.N., Uddin, M.R., Sarker, U.K., Ferdousi,S. and Uddin, M.J. (2017). Allelopathic potential of mustard crop residues on weed management and performance of transplant Aman rice. Journal of the Bangladesh Agricultural University, 15(2):133-139. 
Jabran, K., Mahajan, G., Sardana V. and Chauhan B.S. (2015). Allelopathy for weed control in agricultural systems. Crop Protection, 72:57-65.

Khaliq, A., Matloob, A., Hussain, A., Hussain, S., Aslam, F., Zamir, S.I. and Chattha, M.U. (2015). Wheat residue management options affect crop productivity, weed growth and soil properties in direct-seeded fine aromatic rice. Clean-Soil, Air, Water, 43(8):1259-1265.

Matloob, A., Khaliq, A. Farooq, M. and Cheema Z.A. (2010). Quantification of allelopathic potential of different crop residues for the purple nutsedge suppression. Pakistan Journal of Weed Science Research, 16 (1):1-12

Oad, F., Siddiqui, M. and Buriro, U. (2007). Growth and yield losses in wheat due to different weed densities. Asian journal of plant sciences, 6(1):173-176.

Pramanik, S., Uddin, M.R., Sarker, U.K., Sarkar, D., Ahmed, F. and Alam, M.J. (2019). Allelopathic potential of marshpepper residues for weed management and yield of transplant Aman rice. Progressive Agriculture, 30(4):379-386.

Reddy, K.N. (2001). Effects of cereal and legume cover crop residues on weeds, yield, and net return in soybean (Glycine max). Weed Technology, 15(4):660-668.

Sarker, U.K., Uddin, M.R. and Faruk, G.M. (2020). Weed suppressing ability and performance of common crop residues for sustainable weed management. Journal of Research in Weed Science, 3(3):310-327.

Uddin, M.R. and Pyon, J.Y. (2010). Herbicidal activity of rotation crop residues on weeds and selectivity to crops. Korean Journal of Agricultural Science, 37(1):1-6.

Uddin, M.R., Won, O.J. and Pyon, J.Y. (2010). Herbicidal effects and crop selectivity of sorgoleone, a sorghum root exudate under greenhouse and field conditions. Korean Journal of Weed Science, 30(4):412-420.

Uddin, M.R., Li, X. Won, O.J. Park, S.U. and Pyon, J.Y. (2012).Herbicidal activity of phenolic compounds from hairy root cultures of Fagopyrum tataricum Gaertn. Weed Research, 52(1):25-33.

Uddin, M.R., Park, S.U. Dayan, F.E. and Pyon, J.Y. (2014). Herbicidal activity of formulated sorgoleone, a natural product of sorghum root exudates. Pest Management Science, 70:252-257.

Wazir, I., Sadiq, M. Baloch, M.S. Awan, I.U. Khan, E.A. Shah, I.H. Nadim, M.A. Khakwani, A.A. and Bakhsh, I. (2011). Application of bio-herbicide alternatives for chemical weed control in rice. Pakistan Journal of Weed Science Research, 17(3):245-252.

Won, O.J., Uddin, M.R. and Pyon, J.Y. (2011). Herbicidal activities and crop injury of hairy vetch residues. Korean Journal of Weed Science, 31(2):175-182. 